\title{
Cyclin E involved in early stage carcinogenesis of esophageal adenocarcinoma by SNP DNA microarray and immunohistochemical studies
}

Zhongren Zhou ${ }^{1 *}$, Santhoshi Bandla ${ }^{2}$, Jiqing Ye ${ }^{1}$, Yinglin Xia ${ }^{3}$, Jianwen Que ${ }^{4}$, James D Luketich ${ }^{5}$, Arjun Pennathur ${ }^{5}$, Jeffrey H Peters ${ }^{2}$, Dongfeng $\operatorname{Tan}^{6}$ and Tony E Godfrey ${ }^{7}$

\begin{abstract}
Background: Cyclin $E$ is a cell cycle regulator which is critical for driving G1/S transition. Abnormal levels of cyclin $E$ have been found in many cancers. However, the level changes of cyclin E in esophageal adenocarcinoma and its precancerous lesion have not been well studied. Here, we focus on the gene amplification and expression of cyclin $\mathrm{E}$ in these lesions, and aim to ascertain the relationship with clinicopathological characteristics.

Methods: Genomic DNA was analyzed from 116 esophageal adenocarcinoma and 26 precancerous lesion patients using Affymetrix SNP 6.0 arrays. The protein overexpression of cyclin E was also detected using immunohistochemistry from tissue microarrays containing esophageal adenocarcinoma and precancerous lesions. Patient survival and other clinical data were collected and analyzed. The intensity and percentage of the cyclin $\mathrm{E}$ expressing cells in tissue microarrays were scored by two pathologists. Fisher exact tests and Kaplan-Meier methods were used to analyze data.

Results: By genomic analysis, cyclin E was amplified in 19.0\% of the EAC samples. By immunohistochemistry, high expression of cyclin E was observed in 2.3\% of squamous mucosa tissues, 3.7\% in columnar cell metaplasia, 5.8\% in Barrett's esophagus, $19.0 \%$ in low grade dysplasia, $35.7 \%$ in high grade dysplasia, and $16.7 \%$ in esophageal adenocarcinoma. The differences in cyclin E high expression between neoplastic groups and non-dysplasia groups are statistically significant $(p<0.05)$. The prognosis for patients with high cyclin E expression appeared slightly better than for those with low cyclin E expression although this was not statistically significant $(p=0.13)$.

Conclusions: The expression of cyclin E significantly increases from non-dysplasia esophageal lesion to low and high grade dysplasia, suggesting that cyclin E plays an important role in the early stage of carcinogenesis. Importantly, cyclin E is also amplified and highly expressed in a subset of esophageal adenocarcinoma patients, but this increase is not associated with worse prognosis.
\end{abstract}

Keywords: Esophageal adenocarcinoma, Cyclin E, Amplification, High expression, Barrett's esophagus, SNP DNA microarray, Biomarker, Overall survival

\footnotetext{
* Correspondence: David_zhou@urmc.rochester.edu

'Departments of Pathology and Laboratory Medicine, University of

Rochester, Rochester, 601 Elmwood Ave, Box 626, Rochester, NY 14642, USA

Full list of author information is available at the end of the article
} 


\section{Background}

The incidence of esophageal adenocarcinoma (EAC) has increased approximately $600 \%$ in the US and other Western Countries over the last 30 years [1]. EAC tends to be diagnosed late with most patients in locally advanced or metastatic disease. Consequently, the overall prognosis for patients with EAC is very poor at approximately $15 \%$, with 5-year overall survival. More than 50 percent of patients have either unresectable tumors or radiographically visible metastases at the time of diagnosis [2]. Identification of early biomarkers with high sensitivity and specificity will provide physicians with valuable information for surveillance, diagnosis, prognosis, and possible treatment options for esophageal adenocarcinoma. Previous studies have suggested that esophageal adenocarcinoma develops in the following order: normal esophageal epithelium, reflux esophagitis, Barrett's esophagus (BE), dysplasia, and finally esophageal adenocarcinoma [3]. During these events, a series of genetic and epigenetic aberrations contributes to the carcinogenesis, which will be potential biomarkers for early screening, surveillance and treatment of the dysplasia and adenocarcinoma.

Cyclin E, an activating subunit of cyclin dependent kinase $2(C D K 2)$, is encoded by human cyclin E1 gene (CCNE1) on chromosome 19q12-13. Cyclin E plays a key role to promote $\mathrm{G} 1$ cell cycle transition to $\mathrm{S}$-phase. The oncogenic activity of cyclin $\mathrm{E}$ is involved in multiple functions including a regulatory network comprised CDK inhibitors, the p53 and FBW7 tumor suppressor pathways, signal transduction pathways, controlling cell cycle progression, and microRNAs $[4,5]$. Genetic and pharmacologic targeting of the cyclin E-CDK-2 complex resulted in marked growth inhibition of lung cancer cells [6], suggesting a potential chemotherapeutic approach for lung cancer. In breast cancer, the depletion of cyclin E by siRNA promoted apoptosis of cyclin E overexpressing cells and blocked their proliferation, transformation phenotype and tumor growth in nude mice. Liang and colleagues concluded that cyclin E may serve as a novel and effective therapeutic target [7]. In addition, the amplification and overexpression of cyclin $\mathrm{E}$ have been reported in a variety of cancers including breast [7,8], lung [9], ovarian [10], stomach [11,12], colorectal [13,14], bladder [15], endometrial carcinoma [16] and thyroid [17]. In the esophagus, a few studies found cyclin E amplification and overexpression in esophageal adenocarcinoma and precancerous lesion in small samples [18-21].

The cyclin E expression was first reported in low-grade dysplasia (2/21), high grade dysplasia (3/17), adenocarcinoma (5/35) and Barrett's esophagus (43\%) in 60 samples by an immunohistochemistry [21,22]. Cyclin E gene amplification in esophageal adenocarcinoma was also confirmed in $13.8 \%(9 / 65)$ [19] and $12.6 \%$ (11 of 87) [20] in esophageal adenocarcinoma by quantitative PCR molecular analysis
$[19,20]$. However, the sample size of previous studies is small and the results were not consistent. In addition, the relationship between high expression of cyclin $\mathrm{E}$ or gene amplification and the patient survival is unknown.

In the current study, we (i) used high resolution SNP DNA microarray to study cyclin E amplification in the large scale of esophageal adenocarcinoma and precancerous lesions; (ii) used immunohistochemical method to confirm the high expression of cyclin $\mathrm{E}$ in a larger number of esophageal adenocarcinoma and precancerous lesions; and (iii) studied the association of cyclin E amplification and high expression with patients' overall survival and clinicopathological features.

\section{Methods}

\section{Patients for Affymetrix SNP 6.0 analysis}

Frozen tumors were obtained from 116 patients undergoing esophagectomy at the University of Pittsburgh Medical Center, Pittsburgh, PA between 2002 and 2008. Patient age ranged from 43-88 and the cohort consisted of 95 males and 21 females. Final pathologic stages were stage I (28), stage II (31), stage III (50) and stage IV (7). All tumor specimens were evaluated by a pathologist and were determined to be $>70 \%$ tumor cell representation. Only 112 specimens were used for survival analysis as we excluded 4 peri-operative chemotherapy patients.

Frozen Barrett's esophagus (intestinal metaplasia: $\mathrm{n}=26$ ) and esophageal columnar cell metaplasia (metaplasia without goblet cells; $n=25$ ) biopsy tissues were obtained from patients undergoing endoscopy at the University of Rochester Medical Center from 2008 to 2012. All pathologic diagnoses were evaluated by pathologists. All studies were approved by research subjects review board at University of Pittsburgh and University of Rochester.

\section{Affymetrix SNP 6.0 analysis}

Genomic DNA was isolated using the QiaAmp DNA Mini Kit (Qiagen, CA) and $600 \mathrm{mg}$ was used for labeling and array hybridization at the SUNY Upstate Medical University microarray core facility (Syracuse, NY) using kits and protocols provided by Affymetrix. Data analysis was performed using Nexus 6.0 Copy Number Analysis software (Biodiscovery, Inc. CA). $\log _{2}$ DNA copy number ratios for the tumor and pre-neoplastic samples were generated in reference to a baseline file created using DNA from normal esophageal mucosa from a subset $(n=15)$ of the Pittsburgh patient cohort. Data was segmented using the SNP-Rank segmentation algorithm with a minimum of 8 probe sets and significance threshold of p-value of $10^{-6}$. $\log _{2}$ copy number threshold for gains were set at +0.15 ( $\sim 2.2$ copies $)$ while high level gains were set at $+0.5(\sim 2.8$ copies). More information on this patient cohort and a comprehensive genomic analysis of these tumors is to be published by Dulak et al [23]. Microarray data on this 
cohort has been submitted to the Gene Expression Omnibus (GSE36460) with an online link (http://www.ncbi.nlm. nih.gov/geo/query/acc.cgi?acc=GSE36460).

\section{Construction of tissue microarray}

Tissue microarrays, containing 34 cases of Barrett's esophagus (BE), 81 cases of columnar cell metaplasia (CCM), 86 cases of squamous epithelium (SE), 21 cases of low grade dysplasia (LGD), 14 cases of high grade dysplasia (HGD), and 117 cases of esophageal adenocarcinoma (EAC), were constructed from the representative areas of formalin-fixed specimens collected between 1997-2005 in the Department of Pathology and Laboratory Medicine, University of Rochester Medical Center/Strong Memorial Hospital, Rochester, New York. Five-micron sections were cut from tissue microarrays and were stained with $\mathrm{H} \& \mathrm{E}$ to confirm the presence of the expected tissue histology within each tissue core. Additional sections were cut for immunohistochemistry analysis.

\section{Patients for tissue microarrays}

All 117 patients with EAC used for the tissue microarray construction were treated with esophagectomy at Strong Memorial Hospital/University of Rochester from 1997 to 2005. These patients included 105 males and 12 females. The patients' ages ranged from 34 to 85 years (Table 1 ). The follow-up period after esophagectomy ranged from 0.3 to 142 months with a mean of 39 months.

\section{Immunohistochemistry}

Tissue sections from the tissue microarray were deparaffinized, rehydrated through graded alcohol, and washed with phosphate buffered saline. Antigen retrieval for cyclin $\mathrm{E}$ was performed by heating sections in $99^{\circ} \mathrm{C}$ water bath for 40 minutes. After endogenous peroxidase activity was quenched and nonspecific binding was blocked, ready-to-use mouse monoclonal antibody anti-cyclin $\mathrm{E}$ (Santa Cruz, CA) was incubated at room temperature for 30 minutes. The secondary antibody (Flex HRP) was allowed to incubate for 30 minutes. After washing, sections were incubated with Flex DAB Chromogen for 10 minutes and counterstained with Flex Hematoxylin

\begin{tabular}{|c|c|c|c|c|}
\hline Diagnosis & Total & Female & Male & Age \\
\hline Adenocarcinoma & 117 & 12 & 105 & 65 \\
\hline High grade dysplasia & 14 & 2 & 12 & 67 \\
\hline Low grade dysplasia & 21 & 0 & 21 & 71 \\
\hline Barrett's esophagus & 34 & 4 & 30 & 67 \\
\hline Columnar cell metaplasia & 81 & 7 & 74 & 64 \\
\hline squamous epithelium & 86 & 19 & 67 & 65 \\
\hline
\end{tabular}

for 5 minutes. A colon adenocarcinoma with known cyclin E high expression served as positive control. Negative control was performed by replacing the anti-cyclin E antibody with the normal serum. Several tissue cores were falloff glass during this processing.

\section{Scoring of immunohistochemistry}

All sections were reviewed independently by JY and ZZ blinded to all clinical and pathologic information. Discordant cases were reviewed by both JY and $\mathrm{ZZ}$ and a final consensus was reached. The percentage (0-100\%) of the cells with positive nuclear staining was recorded. The intensity of cyclin E nuclear staining was graded as $0,1+, 2+$, or $3+$. No nuclear stain or positive nuclear stain in less than $10 \%$ was defined as 0 (Figure $1 \mathrm{~A}$ ); weakly nuclear stain in $10 \%$ or more cells was defined as $1+$ (Figure 1B); relatively strong nuclear stain in $10 \%$ or more cells was defined as $2+$ (Figure $1 \mathrm{C}$ ); very strong nuclear stain in $10 \%$ or more cells was defined as $3+$ (Figure 1D). Cyclin E protein was considered highly expressed if $10 \%$ or more of cells stained with a moderate to strong intensity (2+ and $3+$, respectively) (Figure 1 ).

\section{Statistical analysis}

All the descriptive statistics in this study were presented as means. A P-value of less than 0.05 was considered statistically significant. The univariate analysis with cyclin E was conducted first and then followed with a multivariate analysis, including age, gender, and clinical covariates: lymph node metastasis and tumor stage. Chi-square and Fisher exact tests were used as appropriately to compare cyclin E positivity rates from columnar cell mucosa, dysplasia to adenocarcinoma. To evaluate the influence of high expression of cyclin $E$ in esophageal adenocarcinoma, comparative risk analysis using the Kaplan-Meier method cooperated with the log-rank test was performed with cyclin E amplified and non-amplified groups. All the statistical analyses were conducted with SAS 9.3 software (SAS Institute Inc., Cary, NC).

\section{Results}

Genomic analysis of cyclin E amplification

Analysis of 116 EAC specimens using high density copy number microarrays revealed amplification of CCNE1 in $19.0 \%(22 / 116)$ (Figure 2). In this cohort, the median overall survival of patients with CCNE1 amplification was approximately 20 months compared with 25 months for those without amplification $(p=0.22)$. CCNE1 amplification was not observed in Barrett's esophagus (0/26) or columnar cell metaplasia specimens $(0 / 25)$. 


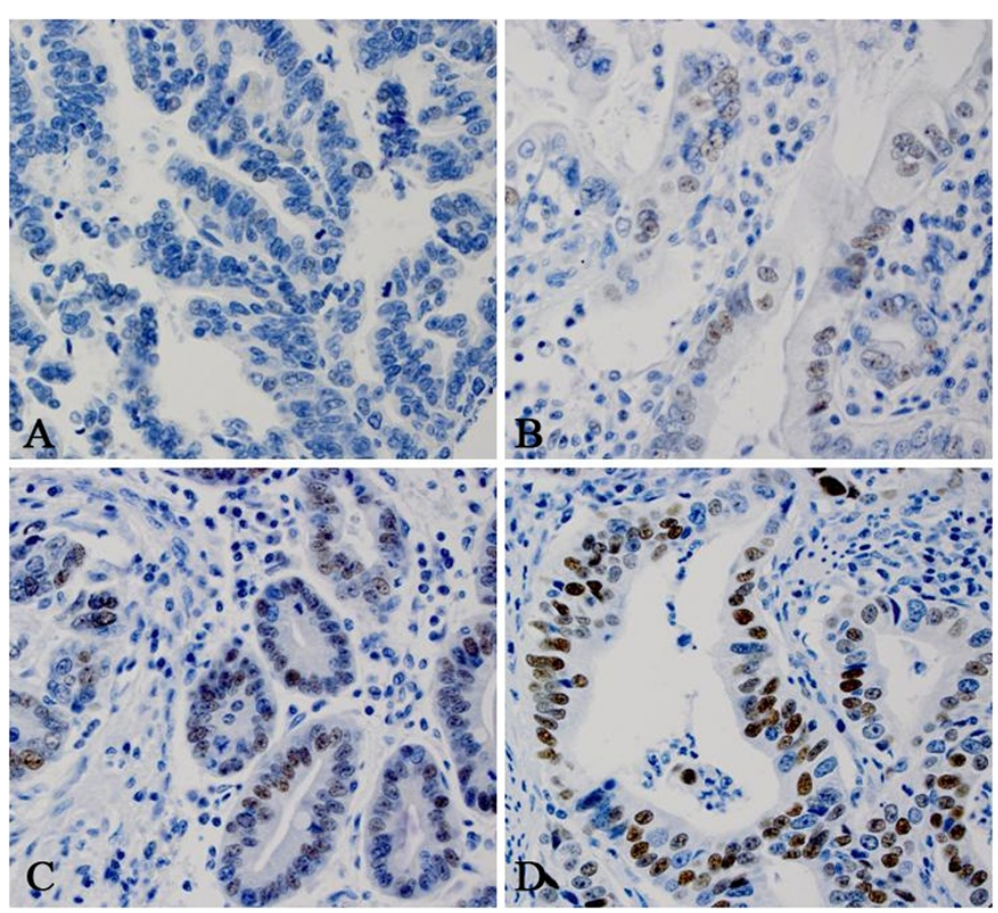

Figure 1 The intensity of cyclin E immunohistochemical study with nuclear staining. A. 0; Negative or very week intensity of cyclin $E$ nuclear stain in one EAC sample; B. 1+: weak intensity of cyclin E nuclear stain in one EAC sample; C. 2+: moderate intensity of cyclin E nuclear stain in one EAC sample; and D. 3+: strong intensity of cyclin E nuclear stain in one EAC sample.

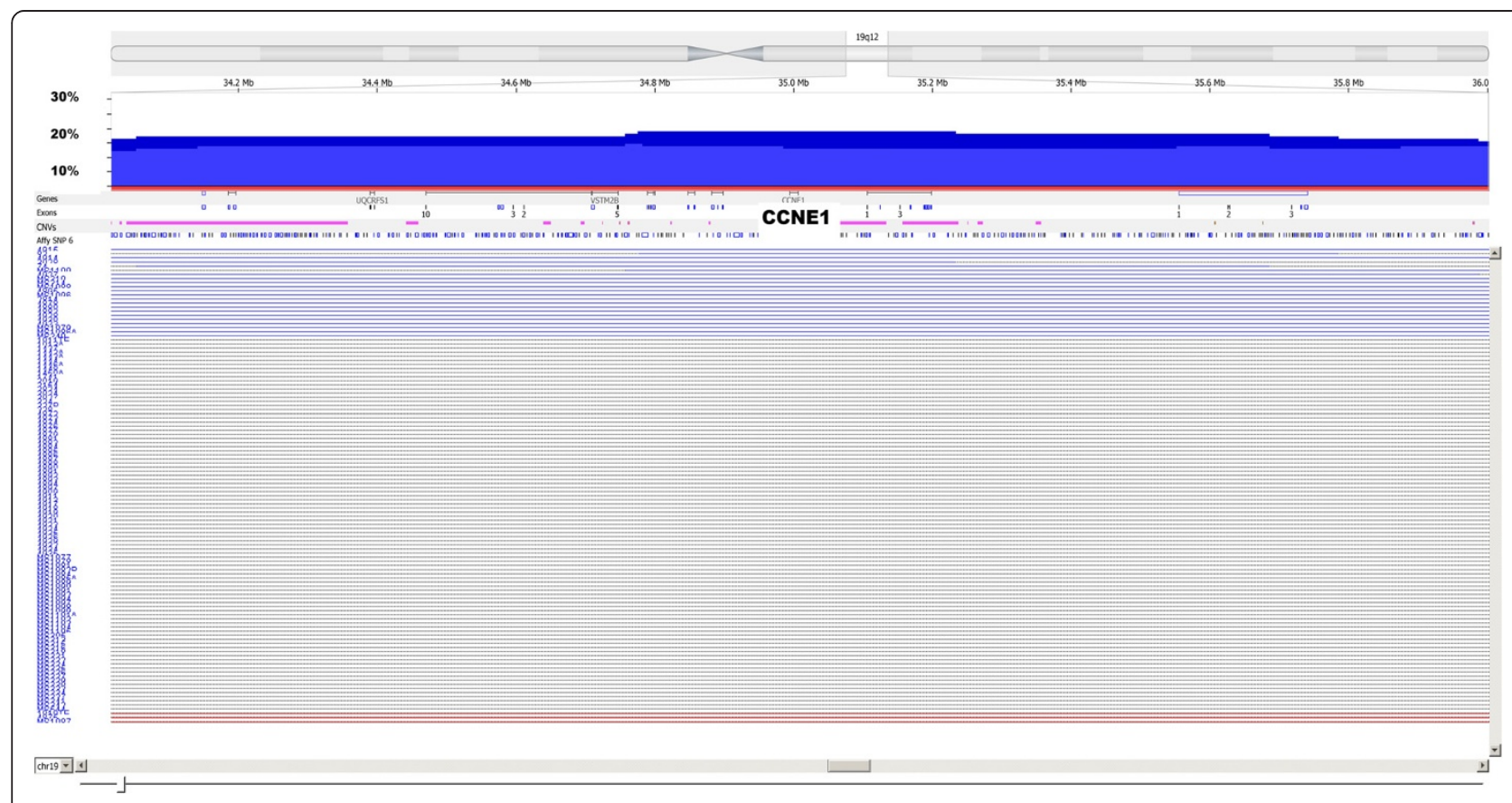

Figure 2 Frequency histogram showing amplification of the CCNE1 locus at chromosome 19q12-13 in 116 esophageal adenocarcinoma samples using high density copy number SNP microarrays. This locus is amplified in 22/116 (19.0\%) cases in this patient cohort, approximately half of which are considered high copy number amplification events. 


\section{Immunohistochemical characteristics and analysis of cyclin E expression}

By immunohistochemical analysis, high expression of cyclin $\mathrm{E}$ was observed in $2.3 \%$ of normal squamous mucosa (2/86), 3.7\% in columnar cell metaplasia (3/81), 5.8\% in Barrett's esophagus (2/34), $19.0 \%$ in low grade dysplasia $(4 / 21)$, and $35.7 \%$ in high grade dysplasia (5/14). In esophageal adenocarcinoma high cyclin E expression was observed in $16.7 \%(19 / 114)$ of cases. This was not statistically significantly different from high grade dysplasia. Qualitatively, we observed that normal squamous mucosa and columnar cell metaplasia usually have weak, focal staining whereas high grade dysplasia or adenocarcinoma have strong, diffuse staining (Table 2 and Figure 3).

Chi-square and Fisher exact tests were used to compare cyclin E percentages among all various histological groups including squamous epithelium, columnar cell mucosa, Barrett's esophagus, low- and high-grade dysplasia, and adenocarcinoma. The differences of cyclin E high expression between all neoplastic groups (including EAC, HGD and LGD) and non-dysplasia groups (including $\mathrm{CCM}$ and $\mathrm{SE})$ are statistically significant $(\mathrm{p}<0.05)$ (Table 3). No significant difference is identified among neoplastic groups. In addition, no significant difference of cyclin E high expression is identified between squamous mucosa and columnar cell metaplasia. Barrett's esophagus group is only significantly different from high grade dysplasia (Table 3).

\section{Survival analysis of cyclin E high expression in EAC}

Kaplan-Meier analysis and the log-rank test were used to calculate the effect of the cyclin $\mathrm{E}$ high expression in patients with EAC on survival. The mean overall survival in the cyclin E high expression group was 42 months, while that in the group without high cyclin E expression was 38 months. The log-rank test showed a trend towards better overall survival in the high-cyclin E group but this was not statistically significance $(p=0.13$, Figure 4$)$.

Multivariate survival analysis of clinical covariates including age, gender, histologic grade, and stage in EAC,

\begin{tabular}{lccc}
$\begin{array}{l}\text { Table } 2 \text { High expression of cyclin E in esophageal } \\
\text { adenocarcinoma, low and high dysplasia, Barrett's }\end{array}$ \\
$\begin{array}{l}\text { esophagus, columnar cell metaplasia and squamous cells } \\
\text { Diagnosis }\end{array}$ & $\begin{array}{c}\text { Non-/low expression } \\
\text { (n; \%) }\end{array}$ & $\begin{array}{c}\text { High expression } \\
\text { (n; \%) }\end{array}$ & Total \\
\hline $\begin{array}{l}\text { squamous epithelium } \\
\text { Columnar cell }\end{array}$ & $84(97.7)$ & $2(2.3)$ & 86 \\
metaplasia & $78(96.3)$ & $3(3.7)$ & 81 \\
Barrett's esophagus & $32(94.2)$ & $2(5.8)$ & 34 \\
Low grade dysplasia & $17(81.0)$ & $4(19.0)$ & 21 \\
High grade dysplasia & $9(64.3)$ & $5(35.7)$ & 14 \\
Adenocarcinoma & $95(83.3)$ & $19(16.7)$ & 114 \\
\hline
\end{tabular}

found that age, differentiation and stage $(p<0.05)$ have strong association with patient survival, but gender $(p=0.66)$ was not significantly associated with patients' survival in EAC.

\section{Correlation of cyclin E high expression and clinicopathological characteristics}

The correlation of high cyclin E expression with clinicopathological features was analyzed. High expression of cyclin $\mathrm{E}$ is not associated with age, gender, stage, differentiation and lymph node metastasis (data not shown).

\section{Discussion}

In this study we found that cyclin E shows a significantly higher frequency of high expression in neoplastic lesions (low- and high-grade dysplasia or adenocarcinoma) compared to non-dysplastic tissues (Barrett's esophagus, columnar cell metaplasia and squamous epithelium). With SNP DNA microarray study, the amplification of cyclin E was also present in esophageal adenocarcinoma, but was not identified in Barrett's esophagus and columnar cell metaplasia. In addition, we found that high expression of cyclin E may be associated with better prognosis although this did not reach statistical significance.

Sarbia et al. first reported that the expression of cyclin $\mathrm{E}$ in esophagus tissues in small samples was present in 0 of $24 \mathrm{SE}(0.0 \%), 2$ of 21 LGD (9.5\%), 3 of 17 HGD (17.6\%), and 5 of 35 CA (14.3\%) [22]. In our study, cyclin E shows similar frequency of high expression in $16.7 \%$ esophageal adenocarcinoma (19/114), but a higher frequency of expression in high grade dysplasia $(35.7 \%)$ and low grade dysplasia (19.0\%) compared to their study. In addition, we found that cyclin $\mathrm{E}$ is highly expressed with lower rates at $5.8 \%$ in Barrett's esophagus (2/34), 3.7\% in columnar cell metaplasia (3/81), and $2.3 \%$ in squamous mucosa $(2 / 86)$. Umansky et al. also reported the expression of cyclin E (43\%), p16 (73\%), p21 (88\%), p27 (95\%), and cyclin D1 (47\%) in Barrett's esophagus, which was down-regulated by acid suppression of proton pump inhibition (PPI). However, no amplification or deletion was identified by Southern blot analysis [21]. This suggests that episodes of acid reflux might trigger proliferation and inhibit programmed cell death signaling pathways. In our study, no amplification was identified in Barrett's esophagus $(0 / 26)$ and columnar cell metaplasia $(0 / 25)$ by SNP DNA microarray method. However, high expression of cyclin E (5.8\%) in BE is significant lower than that in Umansky's study (43\%). The mechanism is unclear how cyclin E is highly expressed in BE and columnar cell metaplasia without the amplification. Cyclin E amplification was observed at $13.8 \%(9 / 65)$ [19] and $12.6 \%$ (11 of 87) [20] in esophageal adenocarcinoma, which is lower than our SNP DNA microarray data (19.0\%). 


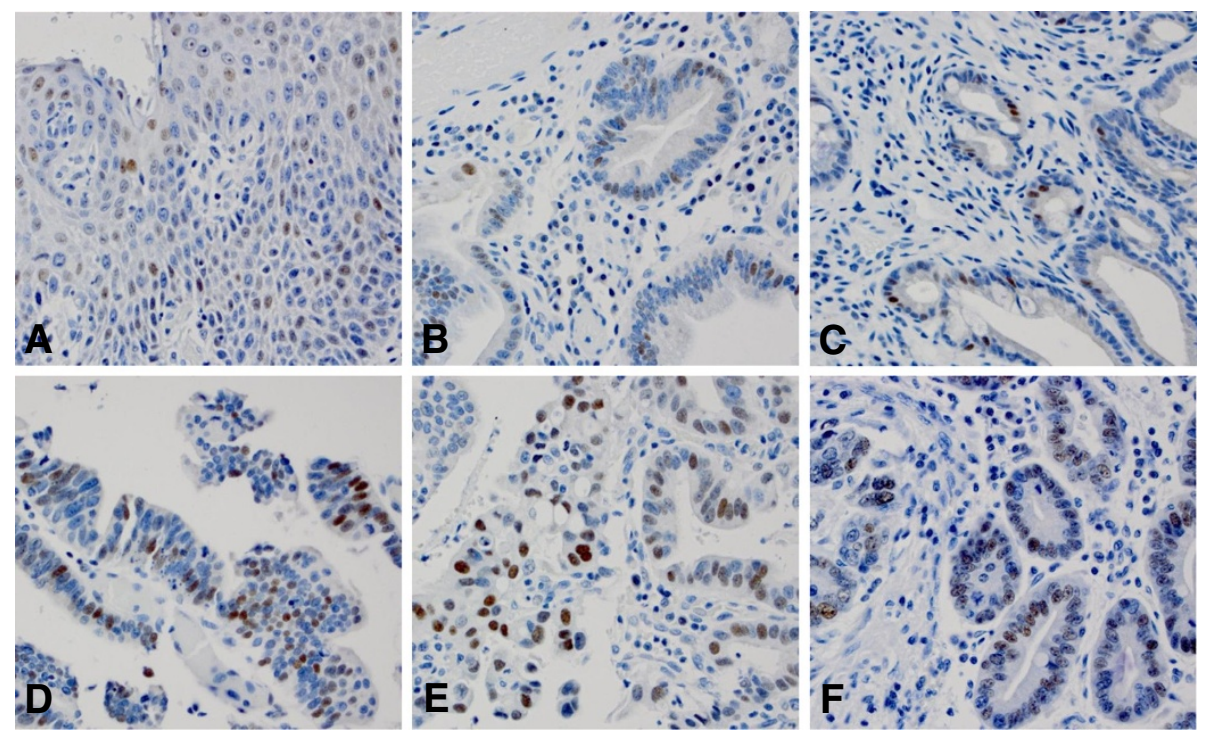

Figure 3 High expression of cyclin $\mathrm{E}$ in various histologic types by immunohistochemical studies. Cyclin $\mathrm{E}$ immunostain shows weakly nuclear stain in squamous mucosa (A), columnar cell metaplasia (B) and Barrett's esophagus (C). Cyclin E shows strong nuclear stain in low grade dysplasia (D), high grade dysplasia (E) and adenocarcinoma (F).

Cyclin E was reported to be expressed in precancerous lesion of colon adenocarcinoma [14,24,25]. Expression of cyclin $\mathrm{E}$ has been shown in $25 \%$ of colorectal adenomas, the most important precursor lesions of colorectal carcinoma [24]. With 1,2-dimethyl-hydrazine dihydrochloride

\section{Table 3 Comparison of the frequency of cyclin E high expression between various groups by Fisher exact test ( $p$ value)}

\begin{tabular}{lll}
\hline Group 1 & Group 2 & $p$ \\
\hline SE & CCM & 0.3060 \\
SE & BE & 0.2496 \\
SE & LGD & $0.0121^{*}$ \\
SE & HGD & $0.0014^{*}$ \\
SE & AC & $0.0011^{*}$ \\
CCM & BE & 0.3119 \\
CCM & LGD & $0.0277^{*}$ \\
CCM & HGD & $0.0014^{*}$ \\
CCM & AC & $0.0051^{*}$ \\
BE & LGD & 0.1158 \\
BE & HGD & $0.0153^{*}$ \\
BE & AC & 0.0890 \\
LGD & HGD & 0.1697 \\
LGD & AC & 0.2192 \\
HGD & AC & 0.0538 \\
\hline
\end{tabular}

*The frequency of cyclin E high expression shows significantly different between these pairs.

$C C M$, Columnar cell metaplasia; $B E$, Barrett's Esophagus; $L G D$, Low grade dysplasia (LGD); HGD, High grade dysplasia; EAC, Esophageal adenocarcinoma; $S E$, Squamous epithelium.
(DMH)-induced rat colon adenocarcinoma, cyclin E expression was detected in $87.5 \%$ of the adenomas and in $92.3 \%$ of the adenocarcinomas [25]. Hur and colleagues also found that cyclin E expression both in the mRNA and protein levels was present in normal colonic mucosa, adenomas and adenocarcinomas. There was a significant difference in the degree of expression of cyclin $\mathrm{E}$ between normal mucosa and adenomas, but there was not a significant difference between adenomas and adenocarcinomas. They indicated that cyclin E plays an important role during the multistage process of rat colon carcinogenesis, especially at a relatively early stage [25]. In human samples, the increase of cyclin $\mathrm{E}$ expression also was reported in colon mucosa. The median of cyclin E expression significantly increased in normal through hyperplastic and adenomatous tissues and slightly decreased in adenocarcinoma of colon samples [14], which confirmed the finding in the rat model and proved that the expression of cyclin $\mathrm{E}$ promoted abnormal proliferation of cells during colorectal carcinogenesis [14]. In the esophagus, our data and previous studies also showed that the high expression of cyclin E significantly increased from non-dysplasia group (normal squamous epithelium, columnar cell metaplasia) to neoplastic group (low and high grade dysplasia). The high expression of cyclin $\mathrm{E}$ reached its peak in high grade dysplasia and decreased in adenocarcinoma. Our findings in the esophagus agree to the previous studies in colon. High expression of cyclin E may play an important role in early stage of carcinogenesis in esophagus and could be a potential targeted marker to early interfere with cancer progress and stratify high risk patients with precancerous lesion for close surveillance. 


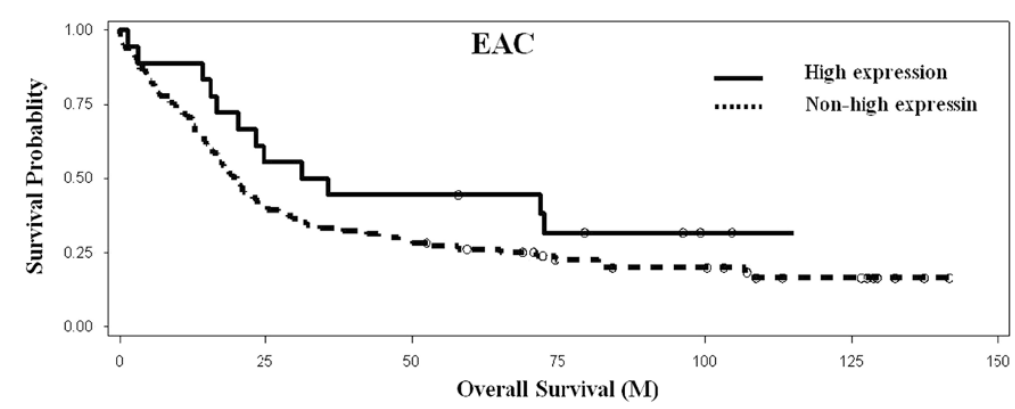

Figure 4 Kaplan-Meier analysis of overall survival associated with high cyclin E expression in esophageal adenocarcinoma. No significant association of overall survival with cyclin E high expression $(p=0.13)$ in 117 EAC patients.

Cyclin E and paired CDK 2 are important antineoplastic targets in oncology. siRNA treatment significantly reduced CCNE1 or cyclin E-CDK-2 complex expression and significantly inhibited cell growth in CCNE1-expressing cells, suggesting that CCNE1-targeted therapy may benefit ovarian, breast and lung cancer patients with CCNE1 amplification [6,7,10]. In addition, cyclin E siRNA synergistically enhanced the cell killing effects of doxorubicin in cell culture and suppressed the tumor growth in mice. They concluded that cyclin E may serve as a novel and effective therapeutic target [7]. Our study showed both amplification and high expression of cyclin $E$ in esophagus precancerous lesion and adenocarcinoma, suggesting the further study of potential effect in the inhibition of cyclin E expression for target therapy of esophageal precancerous lesion.

Amplification and high expression of cyclin E were reported to relate with poor prognosis in many different tumors $[8-10,12,14-16,26]$. In meta-analysis of lung nonsmall cell carcinoma from fourteen studies (2606 cases) [27], cyclin E over-expression was found to be a strong predictor of poor prognosis in lung carcinoma patients (HR: $1.38,95 \%$ CI: 1.07-1.79; P = 0.014). In ovarian cancer, the amplification was identified in 18 (20\%) of 88 ovarian carcinoma, which was significantly correlated with shorter disease-free survival and overall survival [10]. In gastric [11] and colorectal adenocarcinoma [28], overexpression of cyclin E was a potential prognostic markers. It is surprising to find both amplification and high expression of cyclin E in esophageal adenocarcinoma in our study were not significantly associated with patient overall survival, even with a little better overall survival rate with high expression of cyclin E. The controversial data for the prognosis was reported in the colon [29,30], ovary [31], stomach [11,12] and lung [9]. In the esophagus, similar to the cyclin E study, we recently found that HER2 amplification and expression were associated with better but not significantly better prognoses [32], which is confirmed by a Mayo clinical study [33]. They further proved that HER2 positivity was significantly associated with a better survival. Therefore, the function of oncogene may play different roles in various organs or tumors. Furthermore, our findings needs to be confirmed by different studies since the cyclin E expression and amplification are associated with the sensitivity of methods, race of patients, location of tumors and preoperative neoadjuvant therapy.

\section{Conclusions}

The high expression of cyclin E significantly increases from non-dysplasia esophageal lesion, to low and high grade dysplasia. It implies that cyclin E may play an important role in early stage of carcinogenesis and could be a potential marker for a target therapy of precancerous lesion. In addition, the amplification and high expression of cyclin $\mathrm{E}$ are associated with a better prognosis, but not statistically significant.

\section{Competing interests}

The authors declare that they have no competing interests.

\section{Authors' contributions}

ZZ and TG: Designing the project; ZZ: Write the paper. ZZ, TG, JQ and DT: editing the paper and consultation for the project. ZZ and JY: Scoring all IHC slides from TMA; YX: Involving data analysis; TG and SB: Analyzing SNP DNA microarray data; JP, AP, and DT: Collecting the clinicopathological information and tissue. All authors read and approved the final manuscript.

\section{Acknowledgements}

We thank Dr. Jorge Yao for tissue microarray construction. We thank Qi Yang and Loralee McMahon for immunohistochemistry.

All studies were approved by research subjects review board at University of Pittsburgh and University of Rochester. Written informed consent was obtained from the patient for the publication of this report and any accompanying images.

\section{Author details}

'Departments of Pathology and Laboratory Medicine, University of Rochester, Rochester, 601 Elmwood Ave, Box 626, Rochester, NY 14642, USA. ${ }^{2}$ Departments of Surgery, University of Rochester, Rochester, NY, USA. ${ }^{3}$ Biostatistics and Computational Biology, University of Rochester, Rochester, NY, USA. ${ }^{4}$ Biomedical Genetics, University of Rochester, Rochester, NY, USA. ${ }^{5}$ Department of Cardiothoracic Surgery, University of Pittsburgh Medical Center, Pittsburgh, PA, USA. ${ }^{6}$ Department of Pathology, The University of Texas MD Anderson Cancer Center, Houston, TX, USA. 'Department of Surgery, Boston University School of Medicine, Boston, MA, USA. 
Received: 13 March 2014 Accepted: 8 April 2014

Published: 17 April 2014

\section{References}

1. Pohl H, Welch HG: The role of overdiagnosis and reclassification in the marked increase of esophageal adenocarcinoma incidence. J Natl Cancer Inst 2005, 97(2):142-146.

2. Locke GR 3rd, Talley NJ, Fett SL, Zinsmeister AR, Melton LJ 3rd: Prevalence and clinical spectrum of gastroesophageal reflux: a population-based study in Olmsted County, Minnesota. Gastroenterology 1997, 112(5):1448-1456.

3. Enzinger PC, Mayer RJ: Esophageal cancer. N Engl J Med 2003, 349(23):2241-2252.

4. Siu KT, Rosner MR, Minella AC: An integrated view of cyclin E function and regulation. Cell Cycle 2012, 11(1):57-64.

5. Stamatakos M, Palla V, Karaiskos I, Xiromeritis K, Alexiou I, Pateras I, Kontzoglou K: Cell cyclins: triggering elements of cancer or not? World J Surg Oncol 2010, 8:111.

6. Galimberti F, Thompson SL, Liu X, Li H, Memoli V, Green SR, DiRenzo J, Greninger P, Sharma SV, Settleman J, Compton DA, Dmitrovsky E: Targeting the cyclin E-Cdk-2 complex represses lung cancer growth by triggering anaphase catastrophe. Clin Cancer Res 2010, 16(1):109-120.

7. Liang Y, Gao H, Lin SY, Goss JA, Brunicardi FC, Li K: siRNA-based targeting of cyclin E overexpression inhibits breast cancer cell growth and suppresses tumor development in breast cancer mouse model. PLoS One 2010, 5(9):e12860.

8. Keyomarsi K, Tucker SL, Buchholz TA, Callister M, Ding Y, Hortobagyi GN, Bedrosian I, Knickerbocker C, Toyofuku W, Lowe M, Herliczek TW, Bacus SS: Cyclin E and survival in patients with breast cancer. N Engl J Med 2002, 347(20):1566-1575.

9. Kosacka M, Piesiak P, Porebska I, Korzeniewska A, Dyla T, Jankowska R: Cyclin A and Cyclin E expression in resected non-small cell lung cancer stage I-IIIA. In Vivo 2009, 23(4):519-525.

10. Nakayama N, Nakayama K, Shamima Y, Ishikawa M, Katagiri A, lida K, Miyazaki K: Gene amplification CCNE1 is related to poor survival and potential therapeutic target in ovarian cancer. Cancer 2010, 116(11):2621-2634.

11. Jang SJ, Park YW, Park MH, Lee JD, Lee YY, Jung TJ, Kim IS, Choi IY, Ki M, Choi BY, Ahn MJ: Expression of cell-cycle regulators, cyclin E and p21WAF1/CIP1, potential prognostic markers for gastric cancer. Eur J Surg Oncol 1999, 25(2):157-163.

12. Choi MG, Noh JH, An JY, Hong SK, Park SB, Baik YH, Kim KM, Sohn TS, Kim S: Expression levels of cyclin $\mathrm{G} 2$, but not cyclin $\mathrm{E}$, correlate with gastric cancer progression. J Surg Res 2009, 157(2):168-174.

13. Aamodt R, Jonsdottir K, Andersen SN, Bondi J, Bukholm G, Bukholm IR: Differences in protein expression and gene amplification of cyclins between colon and rectal adenocarcinomas. Gastroenterol Res Pract 2009, 2009:285830.

14. Li JQ, Miki H, Ohmori M, Wu F, Funamoto Y: Expression of cyclin E and cyclin-dependent kinase 2 correlates with metastasis and prognosis in colorectal carcinoma. Hum Pathol 2001, 32(9):945-953.

15. del Pizzo JJ, Borkowski A, Jacobs SC, Kyprianou N: Loss of cell cycle regulators $\mathrm{p} 27(\mathrm{Kip} 1)$ and cyclin $\mathrm{E}$ in transitional cell carcinoma of the bladder correlates with tumor grade and patient survival. Am J Pathol 1999, 155(4):1129-1136.

16. Cassia R, Moreno-Bueno G, Rodriguez-Perales S, Hardisson D, Cigudosa JC, Palacios J: Cyclin E gene (CCNE) amplification and hCDC4 mutations in endometrial carcinoma. J Pathol 2003, 201(4):589-595.

17. Wang S, Wuu J, Savas L, Patwardhan N, Khan A: The role of cell cycle regulatory proteins, cyclin $\mathrm{D} 1$, cyclin $\mathrm{E}$, and p27 in thyroid carcinogenesis. Hum Pathol 1998, 29(11):1304-1309.

18. Sarbia M, Bektas N, Muller W, Heep H, Borchard F, Gabbert HE: Expression of cyclin E in dysplasia, carcinoma, and nonmalignant lesions of Barrett esophagus. Cancer 1999, 86(12):2597-2601.

19. Lin L, Prescott MS, Zhu Z, Singh P, Chun SY, Kuick RD, Hanash SM, Orringer MB, Glover TW, Beer DG: Identification and characterization of a 19q12 amplicon in esophageal adenocarcinomas reveals cyclin $\mathrm{E}$ as the best candidate gene for this amplicon. Cancer Res 2000, 60(24):7021-7027.

20. Miller CT, Moy JR, Lin L, Schipper M, Normolle D, Brenner DE, lannettoni $M D$, Orringer $M B$, Beer DG: Gene amplification in esophageal adenocarcinomas and Barrett's with high-grade dysplasia. Clinical Cancer Res 2003, 9(13):4819-4825.

21. Umansky M, Yasui W, Hallak A, Brill S, Shapira I, Halpern Z, Hibshoosh H, Rattan J, Meltzer S, Tahara E, Arber N: Proton pump inhibitors reduce cell cycle abnormalities in Barrett's esophagus. Oncogene 2001, 20(55):7987-7991.

22. Sarbia M, Stahl M, Fink U, Heep H, Dutkowski P, Willers R, Seeber S, Gabbert HE: Prognostic significance of cyclin D1 in esophageal squamous cell carcinoma patients treated with surgery alone or combined therapy modalities. Int J Cancer 1999, 84(1):86-91.

23. Dulak AM, Schumacher SE, Van Lieshout J, Imamura Y, Fox C, Shim B, Ramos AH, Saksena G, Baca SC, Baselga J, Tabernero J, Barretina J, Enzinger PC, Corso G, Roviello F, Lin L, Bandla S, Luketich JD, Pennathur A, Meyerson M, Ogino S, Shivdasani RA, Beer DG, Godfrey TE, Beroukhim R, Bass AJ: Gastrointestinal adenocarcinomas of the esophagus, stomach, and colon exhibit distinct patterns of genome instability and oncogenesis. Cancer Res 2012, 72(17):4383-4393.

24. Yasui W, Kuniyasu H, Yokozaki H, Semba S, Shimamoto F, Tahara E: Expression of cyclin $\mathrm{E}$ in colorectal adenomas and adenocarcinomas: correlation with expression of Ki-67 antigen and p53 protein. Virchows Arch 1996, 429(1):13-19.

25. Hur K, Kim JR, Yoon BI, Lee JK, Choi JH, Oh GT, Kim DY: Overexpression of cyclin D1 and cyclin E in 1,2-dimethylhydrazine dihydrochloride-induced rat colon carcinogenesis. J Vet Sci 2000, 1(2):121-126.

26. Ma Y, Fiering S, Black C, Liu X, Yuan Z, Memoli VA, Robbins DJ, Bentley HA, Tsongalis GJ, Demidenko E, Freemantle SJ, Dmitrovsky E: Transgenic cyclin E triggers dysplasia and multiple pulmonary adenocarcinomas. Proc Natl Acad Sci U S A 2007, 104(10):4089-4094.

27. Huang LN, Wang DS, Chen YQ, Li W, Hu FD, Gong BL, Zhao CL, Jia W: Meta-analysis for cyclin E in lung cancer survival. Clin Chim Acta 2012, 413(7-8):663-668.

28. Corin I, Larsson L, Bergstrom J, Gustavsson B, Derwinger K: A study of the expression of Cyclin $\mathrm{E}$ and its isoforms in tumor and adjacent mucosa, correlated to patient outcome in early colon cancer. Acta Oncol 2010, 49(1):63-69.

29. Bondi J, Husdal A, Bukholm G, Nesland JM, Bakka A, Bukholm IR: Expression and gene amplification of primary (A, B1, D1, D3, and E) and secondary ( $\mathrm{C}$ and $\mathrm{H}$ ) cyclins in colon adenocarcinomas and correlation with patient outcome. J Clin Pathol 2005, 58(5):509-514.

30. loachim E: Expression patterns of cyclins D1, E and cyclin-dependent kinase inhibitors p21waf1/cip1, p27kip1 in colorectal carcinoma: correlation with other cell cycle regulators (pRb, p53 and Ki-67 and PCNA) and clinicopathological features. Int J Clin Pract 2008, 62(11):1736-1743.

31. Bedrosian I, Lu KH, Verschraegen C, Keyomarsi K: Cyclin E deregulation alters the biologic properties of ovarian cancer cells. Oncogene 2004, 23(15):2648-2657.

32. Hu Y, Bandla S, Godfrey TE, Tan D, Luketich JD, Pennathur A, Qiu X, Hicks DG, Peters JH, Zhou Z: HER2 amplification, overexpression and score criteria in esophageal adenocarcinoma. Mod Pathol 2011, 24(7):899-907.

33. Yoon HH, Shi Q, Sukov WR, Wiktor AE, Khan M, Sattler CA, Grothey A, Wu TT, Diasio RB, Jenkins RB, Sinicrope FA: Association of HER2/ErbB2 expression and gene amplification with pathologic features and prognosis in esophageal adenocarcinomas. Clin Cancer Res 2012, 18(2):546-554.

\section{doi:10.1186/1471-230X-14-78}

Cite this article as: Zhou et al:: Cyclin E involved in early stage carcinogenesis of esophageal adenocarcinoma by SNP DNA microarray and immunohistochemical studies. BMC Gastroenterology 2014 14:78. 